\title{
A Novel Technique for Point-wise Surface Normal Estimation
}

\author{
Daniel Barath ${ }^{1,2}$ and Ivan Eichhardt ${ }^{1,2}$ \\ ${ }^{1}$ MTA SZTAKI, Budapest, Hungary \\ ${ }^{2}$ Eötvös Loránd University, Budapest, Hungary
}

Keywords: Surface Normal Estimation, Affine Transformation, Stereo Reconstruction, Oriented Point Cloud, Planar Patch.

Abstract: $\quad$ Nowadays multi-view stereo reconstruction algorithms can achieve impressive results using many views of the scene. Our primary objective is to robustly extract more information about the underlying surface from fewer images. We present a method for point-wise surface normal and tangent plane estimation in stereo case to reconstruct real-world scenes. The proposed algorithm works for general camera model, however, we choose the pinhole-camera in order to demonstrate its efficiency. The presented method uses particle swarm optimization under geometric and epipolar constraints in order to achieve suitable speed and quality. An oriented point cloud is generated using a single point correspondence for each oriented 3D point and a cost function based on photo-consistency. It can straightforwardly be extended to multi-view reconstruction. Our method is validated in both synthesized and real tests. The proposed algorithm is compared to one of the state-of-the-art patch-based multi-view reconstruction algorithms.

\section{INTRODUCTION}

Estimation of surface normal and the related planar patch has been an intensively researched area of computer vision since decades. The aim of this paper is to define a method and describe the theory behind, to estimate planar-like spatial patches (surflets) for each point correspondence in stereo case. We will show that the proposed method can achieve more accurate results in many cases than standard estimation techniques, and it will be a powerful basis for subsequent dense reconstruction algorithms. In our experience, most of the sparse or dense reconstruction methods estimate the spatial positions of the observed points accurately, but they give rough estimations for the patch orientations (the surface normals) most of the time. This motivated our current work.

The algorithm assumes that 2D correspondences (2D point pairs) are already established between images of a stereo pair. The calibration of the cameras in the stereo setup should also be known. We assume that the observed corresponding point pair belongs to the same surflet. After triangulating the position of this observed point from the known $2 \mathrm{D}$ point correspondences, our algorithm further provides information about the underlying surface: the tangent plane at the point. The output of our algorithm is an oriented point cloud, a sparse reconstruction of the scene.

\subsection{Related Work}

Stereo matching and reconstruction methods can be classified into four different classes, based on their applied model for the surface: voxel-based (Faugeras and Keriven, 2002; Pons et al., 2007), deformable polygonal (Zaharescu et al., 2007), depth-map fusion (Strecha et al., 2006) and patch-based (Furukawa and Ponce, 2010; Habbecke and Kobbelt, 2007) methods. Since ours is patch-based, in the rest of the related work we restrict ourselves to this class of methods.

Each patch is built from a local area and the tangent plane of the surface. The tangent plane estimation can be performed directly or indirectly. Direct parametrization of a cost function with the parameters of the tangent plane is an algebraic problem, where the solution is the minimum of the cost function. Indirectly, when a relative (affine) homography is first estimated between the projections of the 3D surflet, the parameters of the tangent plane (and the surface normal) are expressed from this relation (Faugeras and Lustman, 1988). Applied number of views and the nature of the reconstruction (dense or sparse) may vary among the direct or indirect methods.

Such an indirect approach is the work of (Megyesi et al., 2006). Their method requires rectified images to compute the scene structure in two steps. It first generates seed points using affine patch- 
matching, then propagates the estimated surface under a set of conditions. On the seed points they use exhaustive search (ES) to find an optimal 3-DoF affine transformation between patches of the rectified views based on a photo-consistency measure first, then the decomposition of these transformations results in surface normals and disparity. The authors introduce several epipolar geometry-based constraints in order to narrow the search-space. Their normal visibility constraint declares that normals pointing away from the image planes or close to perpendicular to the principal axis can be discarded.

There are several image-based surface normal estimation methods available, such as affine transformation-based technique (Barath et al., 2015) or decomposition of the homography (Faugeras and Lustman, 1988). The study of (Molnár and Chetverikov, 2014) also showed that the surface normal can be expressed directly from the affine homography using the spatial gradients of the $2 \mathrm{D}$ projective functions in the stereo setup. These approaches require full camera calibration and the (affine) homography related to the observed point pair.

Some other methods (Habbecke and Kobbelt, 2006; Furukawa and Ponce, 2010; Lhuillier and Quan, 2005; Vu et al., 2012) can be classified as direct methods for tangent plane estimation. In the work of (Habbecke and Kobbelt, 2006), the authors pose the problem as a plane search in 3D space. This is similar to our approach, but unlike us, they work with matched 2D blobs, and the proposed solution optimizes the plane through 3 parameters. GaussNewton optimization with a photo-consistency-based cost function is used to solve the problem.

From the field of multi-view dense reconstruction we have to mention PMVS (Furukawa and Ponce, 2010). Their method is a patch-based 3-step - match, expand and filter - procedure generating an oriented point cloud (or patches), where the last two steps are repeated $n$ times. Matching is based on minimizing a photometric discrepancy function, therefore, they also optimize in both spatial and image space. After initial matching, for patch optimization they use a gradient method to refine the orientation. In the filtering step the authors use a weak form of regularization, where they apply visibility-based constraints to eliminate incorrect matches and outliers. These constraints show similarities to (Megyesi et al., 2006) and to our approach, but in our method similar constraints are directly applied to the search space, not as a post-processing step. The expansion step can be related to the surface propagation step of (Megyesi et al., 2006). As a final step, of PMVS they also generate a polygonal mesh through Poisson Surface
Reconstruction (Kazhdan et al., 2006) and an Iterative Snapping step. In the latter, they enforce foreground/background segmentation through energy optimization. The weakness of this method comes from initialization of the surface normal (which prefers fronto-parallel patches). As the patch orientation moves away from fronto-parallel, the result of the gradient method gets worse.

There are also a number of multi-step pipelines working on massive number of views (Lhuillier and Quan, 2005; Vu et al., 2012) to achieve high-quality reconstruction. Their first step is usually a crucial one: building an initial sparse or quasi-dense reconstruction of the scene (e.g. oriented point cloud).

Particle Swarm Optimization (PSO) (Kennedy, 2010; Shi and Eberhart, 1998) is a population-based algorithm, designed to find useful solutions for continuous problems in a bounded (or periodic) search space. It is an iterative algorithm trying to find and improve candidate solutions. Multiple particles cooperate seeking one or multiple optima simultaneously. PSO is derivative-free and copes well with noise.

\subsection{Motivation and Goals}

Establishing correspondences between stereo (or multi-view) images is an ambiguous, the reconstruction of the underlying surface an ill-posed problem. There are a number of methods applying constraints and using several views to restrict the problem.

In this paper, we use a direct approach and formulate affine patch-matching in such a way that the 2DoF search space is the same as the parameter space of the surface normal of the corresponding surflet. We preferred using PSO since unlike Gauss-Newton methods, it attempts finding a global optimum without derivatives. However, PSO has - in general - no proof of convergence, in our formulation the quality of the estimated tangent plane is at least as good as if we used a coarse regular grid-based ES. Using the epipolar geometry and direct constraints on the search space, our algorithm opens up a novel way to address high quality reconstruction from photos taken from uncalibrated viewpoints.

This paper and also the algorithm do not deal with full reconstruction. It focuses on individual tangent plane estimation in order to provide a basis for a future multi-view reconstruction algorithm. Potential applications are in the field of 3D reconstruction: generating seed points for surface-propagation, enhancing motion-from-structure in a multi-view setup.

Even though, the commonly used warp function is homography for similar tasks, we chose affine transformation. The benefit of building an affine transfor- 
mation from projection function gradients is, that it is valid for any camera model. We already discussed the simple pinhole-camera based formulation, but e.g. extending the pinhole camera model and its projection function with radial and tangential distortion gives the following advantages.

1. No undistortion of input photos is needed.

2. When warping image patches, no evaluation of camera distortion is needed for each pixel. The affine warp matrix is pre-evaluated, thus transforming the patch is a fast affine image warp.

3. The extension to (not discussed here) omnidirectional cameras is also simple.

Although our affine matching-based algorithm resembles the seed point generation step of different method (Megyesi et al., 2006) with ES, due to our novel formulation of the matching problem, we are able to substitute ES with a search heuristic (PSO), and no rectification of the input images is needed. Remark that the estimation of the affine transformation in their work has 3-DoF since the images are rectified beforehand. To our knowledge, applying PSO to reconstruction problems (Cagnoni, 2008) such as the topic of this paper is new. Also, novel visibility constraints dramatically restrict the search space of PSO.

We performed experiments on synthetic and real data and compared our results to a state-of-the-art method (Furukawa and Ponce, 2010) applied on a stereo image pair as it can be seen in Sec. 4.1.1. Poisson surface reconstruction (Kazhdan et al., 2006) of the output of our method gives satisfactory quality without any post-processing or surface propagation (Megyesi et al., 2006) techniques.

The rest of this study is organized as follows: the Sec. 2 describes the notations used in this paper and the geometric background. Then, in Sec. 3 the core of the proposed algorithm is written in the form of its geometric representation and with novel constraints on the search space, as well. Finally, in Sec. 4 we show that our algorithm works well in synthetic and real tests, and we conclude our research in Sec. 5.

\section{NOTATIONS AND GEOMETRIC BACKGROUND}

In this work matrices are denoted by bold capitals such as $\mathbf{K}$ or $\mathbf{R}$, and vectors are underlined such as $\underline{T}, p, q, \underline{x}$. In addition, the notation $\underline{P}$ usually means a point in $3 \mathrm{D}, p$ is usually a $2 \mathrm{D}$ projection on an image plane and its homogeneous equivalent is $\tilde{p}$.

The projection matrix of the perspective camera

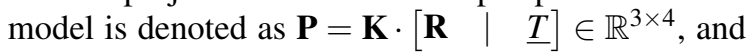

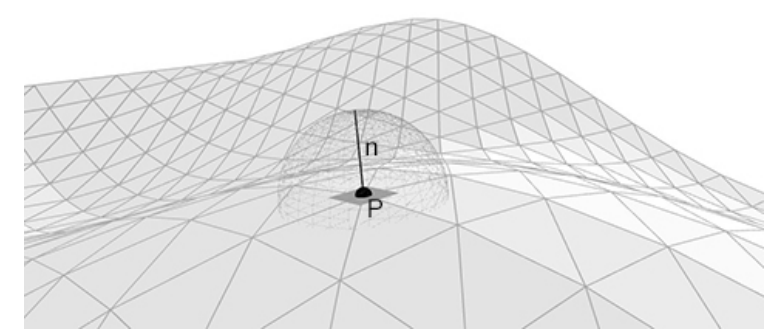

Figure 1: Surface point with normal vector and tangent plane.

constructed using the matrix of intrinsic camera parameters $\mathbf{K} \in \mathbb{R}^{3 \times 3}$, rotation matrix $\mathbf{R} \in \mathbb{R}^{3 \times 3}$ and translation vector $\underline{T}$.

Conversion of a direction $\underline{x} \in \mathbb{R}^{3}$ to Spherical coordinates is expressed as $\operatorname{Sph}(\underline{x})=$ $\left[\arccos \left(\frac{Z}{\|\underline{x}\|}\right) \quad \arctan _{2}(Y, X)\right]^{T}$.

Let $\underline{a}, \underline{b} \in \mathbb{R}^{2}$, such that $\forall i \in\{1,2\}: \underline{a}_{i} \leq \underline{b}_{i}$, then $\operatorname{rect}[\underline{a}, \underline{b}]$ is the minimum (axis-aligned) rectangle containing vectors $\underline{a}$ and $\underline{b}$.

\section{ITERATIVE TANGENT PLANE ESTIMATION}

In this section we show that the normal vector can be robustly estimated from only a single pointcorrespondence, and its local environments on the first, and the second images. Let us denote the proposed method with ITPE in the latter sections.

\subsection{Basis of the Algorithm}

The main idea behind the proposed algorithm is very simple: if a point of a surface is given, the tangent plane of the observed point can be described by the normal vector of the plane as it can be seen in Fig. 1 . It means exactly that the DoF of this plane is two since it can be parametrized by spherical coordinates $(u, v)$ (omitting the third, radial coordinate). Let us denote the stereo camera setup with the projection matrices of the cameras $\mathbf{P}_{1}, \mathbf{P}_{2}$, and the $i^{\text {th }} 3 \mathrm{D}$ point with $\underline{\tilde{P}}^{i}$ in homogeneous form. Its projections on the two cameras are $p_{1}^{i}, p_{2}^{i}$. Now the tangent plane of the observed point can be defined by the point and normal $\underline{n}$. The problem is reduced to a two-dimensional boxconstrained optimization, where the unknown parameters are $u$ and $v$; therefore it always has a solution.

In order to choose the best candidate surface normal we use weighted zero-mean normalized crosscorrelation (ZNCC) (Martin and Crowley, 1995) with Gaussian weights as similarity applied on the pixels 
of the vicinity of the projections of $\underline{P}^{i}$. Although the commonly used technique is to transform the pixels of the patch with the homography, we use its derivative in points $p_{1}^{i}, p_{2}^{i}$ : the affine transformation (Eq. 2). In our experience it gives more accurate results in real scenes (see Table 1) and it is valid for every camera model. Basically, the algorithm solves an optimization problem, maximizing:

$$
\Lambda_{1}(u, v)=\operatorname{ZNCC}\left[\tau_{\underline{p}_{1}} \circ \mathbf{A}(u, v), \tau_{\underline{p}_{2}}\right]
$$

The expression $\tau_{\underline{p}_{j}}$ and operator $\circ$ denotes a local environment of point $\underline{p}_{j}$ (a sub-pixel patch extracted from the image) and the application of an affine transformation matrix (warping the patch), respectively. Function $\mathbf{A}$ in Eq. 2 deduced from the pioneering formulation of (Molnár and Chetverikov, 2014) calculates the affine transformation related to each $(u, v)$ parametrized surface normal:

$$
\mathbf{A}(u, v)=\frac{1}{\left|\nabla x_{1} \underline{n} \nabla y_{1}\right|}\left[\begin{array}{ll}
\left|\underline{n} \nabla y_{1} \nabla x_{2}\right| & \left|\underline{n} \nabla x_{2} \nabla x_{1}\right| \\
\left|\underline{n} \nabla y_{1} \nabla y_{2}\right| & \left|\underline{n} \nabla y_{2} \nabla x_{1}\right|
\end{array}\right]
$$

Using the following derivative forms

$$
\begin{aligned}
\nabla x_{i} & =\frac{1}{s_{i}}\left(\left.\mathbf{P}_{i}\right|_{(1,1: 3)}-\left.x_{i} \cdot \mathbf{P}_{i}\right|_{(3,1: 3)}\right) \\
\nabla y_{i} & =\frac{1}{s_{i}}\left(\left.\mathbf{P}_{i}\right|_{(2,1: 3)}-\left.y_{i} \cdot \mathbf{P}_{i}\right|_{(3,1: 3)}\right) \\
s_{i} & =\left.\mathbf{P}_{i}\right|_{(3,1: 4)} \cdot\left[\frac{P}{1}\right]
\end{aligned}
$$

where $|\underline{a} \underline{b} \underline{c}|$ is the triple product of three vectors $\underline{a}, \underline{b}, \underline{c} \in \mathbb{R}^{3}$ and $\left.\mathbf{P}_{i}\right|_{(k, l: m)}$ denotes the sub-matrix of $\mathbf{P}_{i}$ from row $k$ and columns $l$ to $m$. The surface normal $\underline{n}$ from parameters $u, v$ is as follows:

$$
\underline{n}=\left[\begin{array}{lll}
\cos (u) \sin (v) & \sin (u) \sin (v) & \cos (v)
\end{array}\right]^{T}
$$

To summarize, Eq. 1 maximizes the similarity of the transformed environment in the first image, and the original one in the second image with respect to the surface normal. The problem itself seems to be a simple parameter search, but due the ambiguity of the affine transformation and the deficiencies of photoconsistency several constraints have to be added to the system in order to make it more robust. Remark that due to the symmetrical nature of this problem the proposed cost function can be completed by applying the inverse affine transformation to the second patch.

$$
\Lambda_{2}(u, v)=\operatorname{ZNCC}\left[\tau_{\underline{p}_{1}}, \tau_{\underline{p}_{2}} \circ \mathbf{A}(u, v)^{-1}\right]
$$

Finally, one can compute the best-fitting normal using the following combined cost function:

$$
J^{\prime \prime}=\underset{(u, v)}{\arg \max }\left(\Lambda_{1}(u, v) \Lambda_{2}(u, v)\right)
$$

In our experience Gaussian weighting of the correlation improves the reconstruction.

\subsection{Constraints on the Search-space}

As it can be seen in the previous section, in order to achieve high-quality reconstruction a series of new constraints have to be introduced.

\subsubsection{Visibility constraint}

We can formulate several geometric constraints on our global optimization process, since the 2-DoF search space of the optimal patch is the $(u, v)$ point in spherical coordinates of the corresponding surface normal. The following points introduce (multi-view) visibility-based constraints on the parameter space and the optimization process.

1. Our first boundary constraint utilizes the periodic nature of the spherical coordinates:

$$
\begin{aligned}
& u \in\left[u^{0}-\frac{\pi}{2}, u^{0}+\frac{\pi}{2}\right] \\
& v \in\left[v^{0}-\pi, v^{0}+\pi\right]
\end{aligned}
$$

where $\left(u^{0}, v^{0}\right)=\operatorname{Sph}(\underline{w})$ and $\underline{w}:=-\frac{\mathbf{K}^{-1} \tilde{p}}{\left\|\mathbf{K}^{-1} \underline{\tilde{p}}\right\|_{2}}$, a direction towards the viewpoint. In general,

$$
\left(u_{i}^{0}, v_{i}^{0}\right)=\operatorname{Sph}\left(\underline{w}_{i}\right)=\operatorname{Sph}\left(-\mathbf{R}_{i}^{T} \mathbf{K}_{i}^{-1} \underline{\tilde{p}}_{i}\right)
$$

2. Since a tangent plane not facing the viewer is uninteresting, the search space can be halved:

$$
v \in\left[v_{0}-\frac{\pi}{2}, v_{0}+\frac{\pi}{2}\right]
$$

3. This area can be interpreted as an axis-aligned rectangle in the spherical coordinate system spanning from $\underline{a}^{0}=\left(u^{0}-\frac{\pi}{2}, v^{0}-\frac{\pi}{2}\right)$ to $\underline{b}^{0}=$ $\left(u^{0}+\frac{\pi}{2}, v^{0}+\frac{\pi}{2}\right)$. Let us denote such rectangles as $\operatorname{rect}_{i}:=\operatorname{rect}\left[\underline{a}^{i}, \underline{b}^{i}\right]$ for a given view $i$. Assuming we have multiple views of the patch, taking the intersections of rect $_{i}$ of all $i$ restricts the search space further.

$$
\operatorname{rect}\left[\min _{u, v} \max _{u, v}\right]:=\bigcap_{i}\left(\text { rect }_{i}\right)
$$

The search space can be restricted to the size of a minimal rectangle with both sides equal to $\frac{\pi}{2}$. The search space cannot be restricted more. The following point unfolds this phenomenon while formulating the last geometric constraint.

4. However, if there exists a viewpoint with index $j$, where $\left(u_{j}^{0}, v_{j}^{0}\right) \notin \operatorname{rect}\left[\min _{u, v}, \max _{u, v}\right]$ the search space is empty.

$$
\begin{array}{r}
\exists j:\left(u_{j}^{0}, v_{j}^{0}\right) \notin \operatorname{rect}\left[\min _{u, v}, \max _{u, v}\right] \Longleftrightarrow \\
\exists j \exists i:\left(u_{j}^{0}, v_{j}^{0}\right) \notin \operatorname{rect}_{i} \Longleftrightarrow \\
\exists j \exists i:\left\langle\underline{w}_{j}, \underline{w}_{i}\right\rangle<0
\end{array}
$$


This means that the angle between two view directions is greater than $\frac{\pi}{2}$, implying that one or more viewpoints are outliers.

5. By slightly extending the bounds of rect $_{i}$, the error of the calibration can be accounted for.

\subsubsection{Epipolar Constraint on Affine Transformation}

As it was mentioned before, not the homography is applied on the vicinity of the projected point, but the derived affine transformation. In order to use epipolar-geometry-based constraints a new affine model has to be introduced. Let us denote the affine transformation related to the $i$-th point-pair with

$$
\mathbf{A}^{i}=\left[\begin{array}{cc}
a_{11}^{i} & a_{12}^{i} \\
a_{21}^{i} & a_{22}^{i}
\end{array}\right]=\mathbf{R}_{-\beta}^{i} \cdot \mathbf{W}^{i} \cdot \mathbf{S}^{i} \cdot \mathbf{R}_{\beta}^{i} \cdot \mathbf{R}_{\alpha}^{i}
$$

Matrices $\mathbf{W}^{i}, \mathbf{S}^{i}, \mathbf{R}_{-\beta}^{i}, \mathbf{R}_{\beta}^{i}, \mathbf{R}_{\alpha}^{i}$ are shear, scale and rotations by angles $-\beta, \beta, \alpha$, respectively. $\alpha$ is the angle between the epipolar lines on the first, and the second images that the observed point-pair lies on. $\beta$ is the steepness of the epipolar line on the second image. In summary, this model contains the shear and scale as they are applied parallel to epipolar lines.

It is obvious that due to the nature of perspective projection, mirroring is not allowed $\left(\operatorname{det}\left(\mathbf{A}^{i}\right)>0\right)$. Other restrictions can be made: exclude extremely low scale, and high shear factors as well. In our experience suitable lower bound to the scale and for the upper bound to the shear are 0.2 and 2.0, respectively.

\subsection{Global Optimization}

In our formulation the cost function needs to be minimized on a global scale restricted by the geometric constraints as described in Eq. (10). It is not continuous since we exclude certain affine transformations (see 3.2.2) and is very noisy due to interpolation and the discrete nature of digital images. Performing an ES on the whole parameter space is an option, which gives satisfactory quality, but it is slow.

Our approach was to use PSO, running the iterative algorithm until the average change rate of candidate solutions is less than a threshold $\theta$ in $k$ successive steps. In our experiments we used $10^{-9}$ and 5 , respectively. The swarm is initialized to cover the whole search space like nodes on a uniform grid. Random connections are established between them. This method turned out to be fast and accurate. It is nearly as accurate as an ES-approach and is much faster (see Table 2).

The maximum number of particles used was $n_{\max }=100$ in our tests, but we adaptively decreased

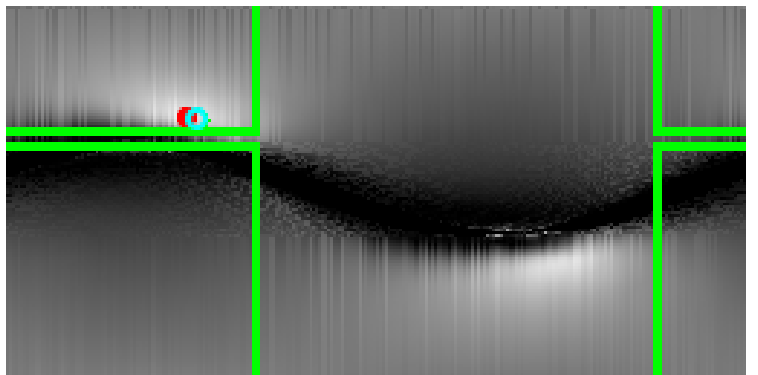

Figure 2: Measurement surface of an experiment on real data. The horizontal and vertical axis are the $u$, and $v$ parameters, respectively. Cropped green rectangle are the area defined by the visibility constraint. Cropping is because of the periodicity of the parameter space. Red and blue dots are the global optimum determined by exhaustive search on the full parameter space and the result of PSO, respectively.

number $n_{\text {opt }}$ as the search area shrinks (see section 3.2.1).

$$
n_{\text {opt }}:=\min \left(4,\left\lceil 100 \frac{\left|\operatorname{rect}\left[\min _{u, v}, \max _{u, v}\right]\right|}{\pi^{2}}\right\rceil\right)
$$

where $\mid$ rect $\mid$ is the area of the rectangle. This gave a mild drop in running times, without losing accuracy.

Fig. 2 shows the measurement surface of an experiment on real data. The horizontal and vertical axis are parameters $u$, and $v$, respectively. Therefore, the coordinates of top-left, and bottom-right points denote $[0,0]^{T}$, and $[2 \pi, \pi]^{T}$, respectively. The cropped green rectangle encapsulates the area defined by the visibility constraint. (Note the periodicity of the parameter space.) Red and blue dots are the global optimum determined by ES on the full parameter space and the result of PSO, respectively. As it is expected, the remaining search space contains the global optimum. The resulting coordinate pair (blue dot) are close to the expected one. There is a gap of invalid (black) values in the middle, which is caused by the constraints based on Eq. 12. Thereby, several values are simply ommited, which are represented by similarity value 0 . It can be seen, that the similarity function is not convex due to the two peaks, and the black constant region. Remark that our preliminary experiments showed, that the ommited area also contains high peaks, even so, the surface normal is invalid there. Unfortunately, gradient descent is not a valid solution for that problem, since the region encapsulated by the visibility rectangle is not convex either.

\section{TESTS}

In this section we show that the proposed method works well on semi-synthesized tests and real-world 
photos. Unless otherwise noted we used $s=70$ for 2D patch sizes and $\sigma=\frac{s}{2}$ for the Gaussian.

\subsection{Semi-synthesized Tests}

Three type of well-textured synthetic scenes were generated using Processing ${ }^{1}$ : a unit sphere, a unitedge cube, and a complex scene. This scene consists of two perpedicual planes, a cylinder, a sphere, and a parametric surface. The intrinsic and the extrinsic parameters of the camera setups were known, as well. We took screenshots of the scenes from different viewpoints (see Fig. 3). In order to get feature points, ASIFT feature matcher (Yu and Morel, 2009) was applied. Then the proposed algorithm ran on each point pair, in order to compute the surflets. The computed error value is the average angular error of the surface normals w.r.t. the ground truth ones.

Fig. 3 shows two views of "Sphere", "Cube", and "Complex" test scenes. ${ }^{2}$ For the sake of comparison, we reconstructed the scene by PMVS (Furukawa and Ponce, 2010) and the standard LS Plane (Hoppe et al., 1992) algorithm was also applied, in order to estimate surface normals from the raw point clouds.

In Table 1, four methods are evaluated: ITPEPSO, ITPE-ES, PMVS and LS Plane. It is visible that our method (ITPE-PSO) achieves smaller than $6.5^{\circ}$ average angular error in every test case, and the median errors are also below $3.5^{\circ}, 1.2^{\circ}$, and $3.5^{\circ}$ for the sphere, cube and complex scenes, respectively. This means that in the case of the cube, half of the surface normals are closer to the ground truth than $1.2^{\circ}$. It is also shown that the results of the rival methods are approximately three, ten, and two times less accurate in the three cases. In Table 2 processing times of ITPEPSO, ITPE-ES methods are shown. It is apparent that ITPE-PSO gives almost the same results as ITPE-ES (Table 1), even so it is is ten times faster (Table 2).

Synthesized tests show that the proposed algorithm works well and its processing time is low. As it can be seen in Table 1, the average angular error of the estimated surface normal in sphere and cube tests are $5.5^{\circ}$ and $2.09^{\circ}$, respectively. This result is considerable given per-point estimation.

\subsubsection{Free-form Surfaces}

The proposed algorithm is also applied to real photos we acquired by handheld cameras. We assume

\footnotetext{
${ }^{1}$ https://processing.org/

${ }^{2} 3 \mathrm{D}$ reconstruction results are visualized in (Cignoni et al., 2008). The test environment was a notebook with an Intel(R) Core(TM) i7-3610QM CPU at $2.30 \mathrm{GHz}$, with 8 cores and $8192 \mathrm{MB}$ of RAM.
}

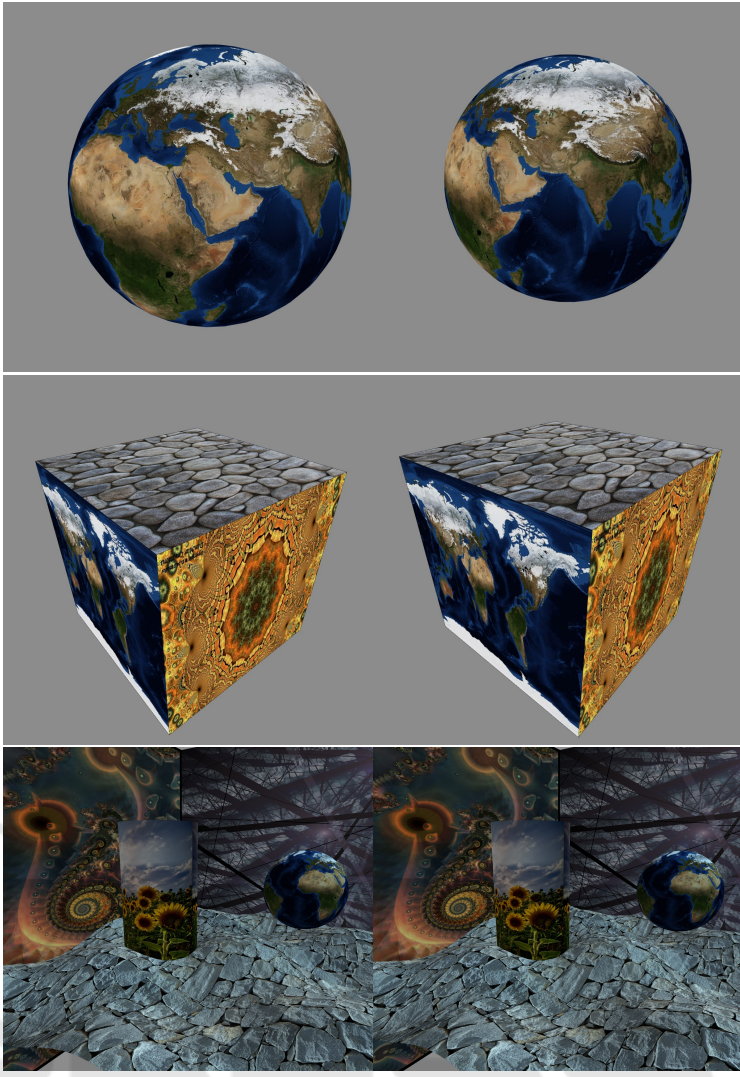

Figure 3: Input stereo image pairs of the synthetic tests. The first, second and third rows show the input of test cases "Sphere", "Cube" and "Complex", respectively.

that the intrinsic parameters are known. The relative pose of the setup is recovered (Bradski et al., 2000) from the Essential matrix, which is calculated from the Fundamental matrix $(\mathbf{F})$. $\mathbf{F}$ is estimated from corresponding ASIFT (Morel and Yu, 2009) point pairs.

Then ITPE-PSO method was applied to each point pair. In order to validate the results, we performed Poisson reconstruction (Kazhdan et al., 2006) of the $3 \mathrm{D}$ point clouds and the related normals.

Fig. 4 consists of photos of a textured bear (honey bottle) with high curvatures. The first two images are the stereo image pair that was used during the normal reconstruction. The last two images are two views of the reconstructed surface. As it can be seen our algorithm accurately follows the shape of the observed object even though the estimation is point-wise, it takes only the local features into consideration. The quality of the reconstruction is the best observed around the nose of the bear from a side-wise view. The result is much better than MeshLab LS Plane implementation even though the curvature is high.

For the sake of comparison, we used $\mathrm{PMVS}^{3}(\mathrm{Fu}-$

\footnotetext{
${ }^{3} \mathrm{http}: / / \mathrm{cc} w u . \mathrm{me} / \mathrm{vsfm}$ (application available online)
} 
Table 1: Estimation results with $\sigma=50$ and window size is $100 \mathrm{px}$.

\begin{tabular}{|c|c|c|c|c|c|c|}
\hline & \#points & Avg. ang. err. & Med. ang. err. & Avg. dist. err. & Med. dist. err. \\
\hline & ITPE-PSO & \multirow{3}{*}{9492} & $5.5225^{\circ}$ & $3.4042^{\circ}$ & \multirow{3}{*}{0.0310} & \multirow{3}{*}{0.0321} \\
\hline \multirow{3}{*}{ 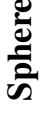 } & ITPE-ES & & $\mathbf{5 . 5 0 2 1}^{\circ}$ & $3.3994^{\circ}$ & & \\
\hline & LS Plane & & $30.5298^{\circ}$ & $22.1780^{\circ}$ & & \\
\hline & PMVS & 12658 & $16.6978^{\circ}$ & $9.4666^{\circ}$ & 0.0416 & 0.0433 \\
\hline \multirow{4}{*}{ שٌ } & ITPE-PSO & \multirow{3}{*}{9960} & $2.0883^{\circ}$ & $1.1481^{\circ}$ & \multirow{3}{*}{0.0581} & \multirow{3}{*}{0.0585} \\
\hline & ITPE-ES & & $2.0767^{\circ}$ & $1.1352^{\circ}$ & & \\
\hline & LS Plane & & $25.1969^{\circ}$ & $29.6932^{\circ}$ & & \\
\hline & PMVS & 13376 & $24.6775^{\circ}$ & $21.9029^{\circ}$ & 0.0908 & 0.0907 \\
\hline \multirow{4}{*}{ 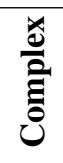 } & ITPE-PSO & \multirow{3}{*}{15343} & $6.3756^{\circ}$ & $3.4440^{\circ}$ & \multirow{3}{*}{0.0181} & \multirow{3}{*}{0.0158} \\
\hline & ITPE-ES & & $6.3461^{\circ}$ & $\mathbf{3 . 4 2 8 0}^{\circ}$ & & \\
\hline & LS Plane & & $22.0623^{\circ}$ & $11.0703^{\circ}$ & & \\
\hline & PMVS & 47114 & $12.0152^{\circ}$ & $9.7374^{\circ}$ & 0.0272 & 0.0283 \\
\hline
\end{tabular}

Table 2: Per-point processing times of ITPE-PSO and ITPE-ES methods (window size is $100 \mathrm{px}$ ).

\begin{tabular}{|c|c|c|}
\hline & Sphere & Cube \\
\hline ITPE-PSO & $\mathbf{0 . 0 2 6 5}$ sec & $\mathbf{0 . 0 2 8 3 ~ s e c}$ \\
\hline ITPE-ES & $0.1884 \mathrm{sec}$ & $0.2035 \mathrm{sec}$ \\
\hline
\end{tabular}

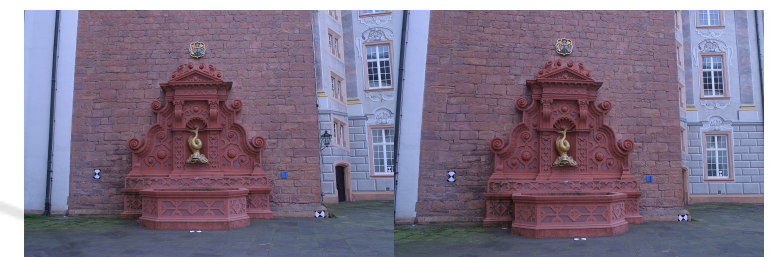

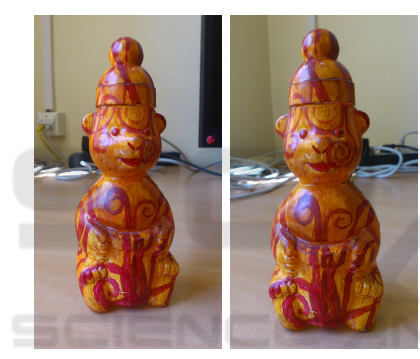

Figure 4: The first two images is the stereo image pair taken from the observed honey bear. The last two images are views of the Poisson reconstruction applied to the resulting oriented point cloud, respectively.

rukawa and Ponce, 2010) to reconstruct the scene using an image pair from dataset fountain-P11 ${ }^{4}$. Then the same stereo pair was processed by our method, as well. Surflets were estimated in both cases, then Poisson reconstruction was applied to the resulting point clouds using the same parameter setup.

Fig. 5 shows Poisson reconstructions applied to the output of the methods. The first row consists of the stereo image pair which was used. The second and third rows consist of the results of PMVS and ITPE from different viewpoints, respectively. It is obvious that our method gives much more sophisticated result using the same parameter setup. Remark that PMVS implements dense reconstruction and surface propagation, as well, by contrast our proposed method does not. Even so our results approximate the original surface significantly better using the same input.

\footnotetext{
${ }^{4}$ http://cvlabwww.epfl.ch/data/multiview/
}

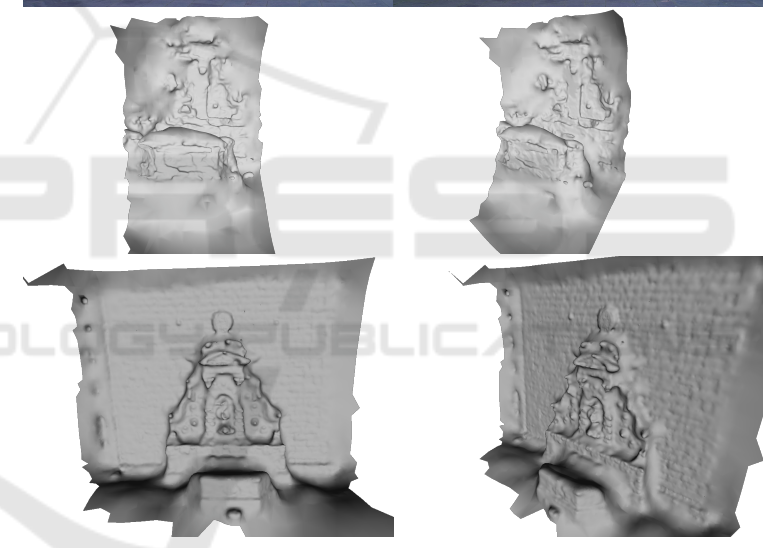

Figure 5: The first, second and the third rows consist of stereo image pairs taken from Fountain dataset, Poisson reconstruction applied on the result of PMVS from two viewpoints and the reconstruction of ITPE-PSO from two viewpoints, respectively.

\section{CONCLUSION}

This paper was motivated by the imprecision of the normal vectors estimated by state-of-the-art reconstruction algorithms. As it is shown the proposed method can estimate more accurate tangent planes than the rival algorithms and the same accuracy of the point locations. The benefit of using affine transformation instead homography is that it makes the proposed theory applicable to general camera model. Moreover, no image undistortion needed.

Compared to other methods (Furukawa and 
Ponce, 2010; Hoppe et al., 1992; Vu et al., 2012; Megyesi et al., 2006; Lhuillier and Quan, 2005) the novelty of the proposed algorithm is threefold:

1. As a theoretical contribution: the search space is narrowed by novel epipolar and geometry-based constraints. It is mathematically ensured that the new search space still contains the optimal solution. These proposed constraints can be extended to multi-view reconstruction straightforwardly.

2. Particle Swarm Optimization makes global optimum available with suitable speed. The proposed method is well-parallelizable, with its per-point processing time is below $0.03 \mathrm{sec}$. Therefore, a good GPU implementation could make it real-time capable.

3. It is applicable to various types of cameras, such as the perspective and omni-directional ones.

We believe that the proposed method is a powerful tool to be used for sparse reconstruction and provides a good base for future multiple-view methods.
Kennedy, J. (2010). Particle swarm optimization. In Encyclopedia of Machine Learning, pages 760-766.

Lhuillier, M. and Quan, L. (2005). A quasi-dense approach to surface reconstruction from uncalibrated images. Pattern Analysis and Machine Intelligence, IEEE Transactions on, 27(3):418-433.

Martin, J. and Crowley, J. L. (1995). Comparison of correlation techniques. In International Conference on Intelligent Autonmous Systems, Karlsruhe (Germany), pages 86-93.

Megyesi, Z., Kós, G., and Chetverikov, D. (2006). Surface normal aided dense reconstruction from images. In Proceedings of Computer Vision Winter Workshop. Telc:[sn]. Citeseer.

Molnár, J. and Chetverikov, D. (2014). Quadratic transformation for planar mapping of implicit surfaces. Journal of mathematical imaging and vision, 48(1):176184.

Morel, J.-M. and Yu, G. (2009). Asift: A new framework for fully affine invariant image comparison. SIAM Journal on Imaging Sciences, 2(2):438-469.

Pons, J.-P., Keriven, R., and Faugeras, O. (2007). Multiview stereo reconstruction and scene flow estimation with a global image-based matching score. International Journal of Computer Vision, 72(2):179-193.

Shi, Y. and Eberhart, R. (1998). A modified particle swarm optimizer. In Evolutionary Computation Proceedings, 1998. IEEE World Congress on Computational Intelligence., The 1998 IEEE International Conference on, pages 69-73. IEEE.

Strecha, C., Fransens, R., and Van Gool, L. (2006). Combined depth and outlier estimation in multi-view stereo. In CVPR'06 IEEE Computer Society Conference on, volume 2, pages 2394-2401. IEEE.

Vu, H.-H., Labatut, P., Pons, J.-P., and Keriven, R. (2012). High accuracy and visibility-consistent dense multiview stereo. Pattern Analysis and Machine Intelligence, IEEE Transactions on, 34(5):889-901.

Yu, G. and Morel, J.-M. (2009). A fully affine invariant image comparison method. In ICASSP 2009. IEEE International Conference on, pages 1597-1600. IEEE.

Zaharescu, A., Boyer, E., and Horaud, R. (2007). Transformesh: a topology-adaptive mesh-based approach to surface evolution. In $A C C V^{\prime} 07$, pages 166-175. Springer. 